\title{
Influence of the oncolytic parvovirus H-I, CTLA-4 antibody tremelimumab and cytostatic drugs on the human immune system in a human in vitro model of colorectal cancer cells
}

\author{
This article was published in the following Dove Press journal: \\ OncoTargets and Therapy \\ 19 August 2013 \\ Number of times this article has been viewed
}

\author{
Bernd Heinrich* \\ Katrin Goepfert* \\ Maike Delic \\ Peter R Galle \\ Markus Moehler \\ University Medical Center of the \\ Johannes Gutenberg University Mainz, \\ Ist Department of Internal Medicine, \\ Langenbeckstrasse, Mainz, Germany \\ *These authors contributed equally \\ to this work
}

\begin{abstract}
Introduction: Tumor-directed and immune-system-stimulating therapies are of special interest in cancer treatment. Here, we demonstrate the potential of parvovirus $H-1$ (H-1PV) to efficiently kill colorectal cancer cells and induce immunogenicity of colorectal tumors by inducing maturation of dendritic cells (DCs) alone and also in combination with cytostatic drugs in vitro. Using our cell culture model, we have additionally investigated the effects of anti-CTLA-4 (cytotoxic T-lymphocyte-associated antigen 4) receptor antibody tremelimumab on this process.
\end{abstract}

Materials and methods: Colon carcinoma cell lines were treated with different concentrations of cytostatic drugs or tremelimumab or were infected with H-1PV in different multiplicities of infection (MOIs), and viability was determined using MTT assays. Expression of CTLA-4 in colon carcinoma cell lines was measured by FACScan ${ }^{\mathrm{TM}}$. For the coculture model, we isolated monocytes using adherence, and differentiation into immature DCs (iDCs) was stimulated using interleukin-4 and granulocyte-macrophage colony-stimulating factor. Maturation of iDCs into mature DCs (mDCs) was induced by a cytokine cocktail. SW480 colon carcinoma cells were infected with H-1PV or treated with cytostatic drugs. Drug treated and H-1PV-infected SW480 colon carcinoma cells were cocultured with iDCs and expression of maturation markers was measured using FACScan ${ }^{\mathrm{TM}}$. Cytokine measurements were performed using enzyme-linked immunosorbent assay.

Results: Colon carcinoma cells SW480 were potently infected and killed by H-1PV. CTLA-4 expression in SW480 cells increased after infection with H-1PV and also after treatment with cytostatic drugs. Tremelimumab had no influence on viability of the colon carcinoma cell line. There was no maturation of iDCs after coculture with SW480; instead, H-1PV-infected or drug pretreated SW480 induced maturation. Cytokine production was higher for H-1PV-infected cells but was not significantly enhanced by tremelimumab treatment alone or in combination. Addition of tremelimumab did not interfere with the maturation process as measured by markers of maturation as well as by determination of cytokine levels.

Conclusion: By enhancing both cell death and immunogenicity of tumors, H-1PV is of special interest for tumor-directed therapy. These features make it a promising candidate for clinical application in human colorectal cancer. As tremelimumab does not significantly interfere with this process, an interesting therapeutic combination of active enhancement of tumor immunogenicity and independent masking of the CTLA-4 silencing process on tumor cells is highlighted.

Keywords: dendritic cells, SW480

\section{Introduction}

A targeted, tumor-directed and immune-system-stimulating therapy is of special interest in cancer treatment. ${ }^{1,2}$ We and other groups have previously demonstrated the oncolytic 
and immunogenic potential of oncolytic viruses ${ }^{3-5}$ as one option towards this goal. Taking into account the positive results for melanoma and hepatocellular carcinoma cells, ${ }^{6,7}$ and knowing that Parvoviridae are able to target colorectal cancer cells, ${ }^{8}$ we aimed to investigate the parvovirus $H-1$ (H-1PV) infection of colorectal cancer cells. H-1PV has been shown to exert selective cytotoxic effects and shows potential to increase maturation of dendritic cells (DCs). ${ }^{9}$

DCs play an important role in anticancer immunity, especially by cross-talking and interacting with cytotoxic $\mathrm{T}$ cells ${ }^{10,11}$ and with their function as antigen presenting cells. ${ }^{12}$ On the other hand, expression of cytotoxic T-lymphocyteassociated antigen 4, CD-152 (CTLA-4), on the surface of human tumor cells is a strategy to circumvent the human immune system. ${ }^{13,14}$ CTLA-4 is a member of the immunoglobulin superfamily, which is expressed on the surface of activated $T$ helper $\left(T_{h}\right)$ cells and transmits an inhibitory signal to $T$ cells. However, tumor cells, including colorectal cancer cells, often express CTLA-4 on their surface to generate an environment that leads to immune escape and saves tumor cells from being attacked by activated effector cells of the immune system. ${ }^{1}$

Following the idea of stimulating immune defense mechanisms, the focus in the last few years was on molecules like CTLA-4, the B7 family, and programmed cell death 1 (PD-1). ${ }^{15-17}$ Tremelimumab (formerly ticilimumab, CP-675,206; Pfizer, Inc, New York, NY, USA) is a fully human monoclonal antibody specific for CTLA-4. Blocking the CTLA-4 negative costimulatory receptor with tremelimumab results in immune activation. ${ }^{16}$ With the pro-immunogenic effects of $\mathrm{H}-1 \mathrm{PV}$ in mind and the idea of overcoming the immune-escaping effects of CTLA-4 expressing colorectal carcinoma cell lines, ${ }^{1}$ combination therapy of these two agents is of interest.

In the case of melanoma cells, tremelimumab is well analyzed, ${ }^{18,19}$ but little is known for ex vivo models of colorectal cancer. As CTLA-4 is described to be expressed on colorectal cancer cells and also to trigger apoptosis, ${ }^{13}$ we investigated the influence of tremelimumab treatment on cell-viability and CTLA-4 expression, both alone and in combination with clinically relevant cytostatic drugs 5-fluorouracil, oxaliplatin, and irinotecan (Pfizer) as well as H-1PV. As CTLA-4 is also of importance for maturation and antigen presentation of DCs, ${ }^{12,20}$ we measured effects of tremelimumab and H-1PV on cytokine levels including combinations of cytostatic drugs, as combined therapy strategies were described to gain pronounced immunostimulation via DC maturation. .,21 $^{2}$

\section{Materials and methods Human colon carcinoma cells and human immune cells}

Human colon carcinoma cell lines SW480, Caco-2, HCT116, and HT29 (all Leibniz Institute DSMZ-German Collection of Microorganisms and Cell Cultures, Braunschweig, Germany;

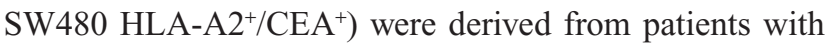
human colon carcinoma. SW480, HCT116, and HT29 cells were cultured in RPMI (Roswell Park Memorial Institute) medium (Gibco ${ }^{\circledR}$; Life Technologies, Carlsbad, CA, USA) with $10 \%$ fetal calf serum (FCS) (FCS; PAA Laboratories $\mathrm{GmbH}$, Cölbe, Germany) and 1\% penicillin/streptomycin (Gibco ${ }^{\circledR}$; Life Technologies). Caco-2 cells were cultured in RPMI medium with $20 \%$ FCS and $1 \%$ penicillin/ streptomycin. Cells were cultivated in culture flasks at $5 \%$ $\mathrm{CO}_{2}$ and at $37^{\circ} \mathrm{C}$.

Monocytes were isolated by adherence from HLA-A2 positive human buffy coats. Monocytes were treated with $500 \mathrm{U}$ interleukin-4 (IL-4; ImmunoTools GmbH, Friesoythe, Germany) and $500 \mathrm{U}$ granulocyte-macrophage colony-stimulating factor (GM-CSF; Bayer HealthCare Pharmaceuticals, Montville, NJ, USA) for 6 days to produce immature DCs (iDCs). Treatment with $0.01 \mu \mathrm{g} / \mathrm{mL}$ tumor necrosis factor alpha (TNF- $\alpha$ ), IL-6, IL-1- $\beta$ (all Miltenyi Biotec, Bergisch Gladbach, Germany), plus $1 \mu \mathrm{g} / \mathrm{mL}$ prostaglandin E2 (PGE2) (Sigma-Aldrich, St Louis, MO, USA) produced mature dendritic cells (mDCs).

\section{Cell treatment and MTT viability assay}

For MTT (3-[4,5-Dimethylthiazol-2-yl]-2,5-diphenyltetrazoliumbromide) assay cells were seeded in 96-well plates for cytostatic drugs and tremelimumab, and in 6-well plates for $\mathrm{H}-1 \mathrm{PV}$ infection.

For single treatment in the MTT assay, serial dilutions of cytostatic drugs were generated and added to the different cell lines. Influence of 5-fluorouracil, irinotecan, and oxaliplatin on cell viability was tested for by applying concentrations of $0-500 \mu \mathrm{g} / \mathrm{mL}$. Influence of tremelimumab on cell viability was measured by application of concentrations of $0-240 \mu \mathrm{g} / \mathrm{mL}$. Cells were incubated for 24 hours, 48 hours, and 72 hours. For infection with H-1PV, cell medium was removed and cells were incubated with a minimal amount of complete medium for 1 hour. Subsequently, an appropriate volume of medium was added and cells were incubated for up to 5 days post infection. Multiplicity of infection (MOI) was chosen from 1 to 40 plaque-forming units (PFU)/cell. 
MTT was added and, after dissolving the produced purple formazan with sodium dodecyl sulfate (SDS), absorption was measured at $562 \mathrm{~nm}$ by a spectrophotometer (Bio Tek Instruments, Winooski, VT, USA).

\section{CTLA-4 expression}

From the MTT assay, IC-20 (inhibitory concentration) was calculated for treatment with 5-fluorouracil, oxaliplatin, and irinotecan, and a middle incubation time of 48 hours was chosen to treat cell lines. A MOI of $20 \mathrm{PFU} /$ cell was chosen for $\mathrm{H}-1 \mathrm{PV}$ infection for 5 days.

For extracellular expression measurement, cells were harvested and incubated with phycoerythrin (PE) labeled anti-CTLA-4 antibodies (R\&D Systems, Minneapolis, MN, USA). For intracellular measurement, cells were harvested and first treated with paraformaldehyde and methanol to destroy the cell membranes. Then cells were incubated with anti-CTLA-4 antibodies. CTLA-4 expression was documented by flow cytometric analysis with a FACScan ${ }^{\mathrm{TM}}$ (BD Biosciences, San Jose, CA, USA).

\section{Coculture model and expression of activation and maturation markers on DCs}

For coculture model, SW480 cells were seeded in 6-well plates and treated as described. For comparable treatment, IC-20 and an incubation period of 48 hours were chosen for 5-fluorouracil, irinotecan, and oxaliplatin, and calculated from MTT assays. Tremelimumab was used at a middle concentration of $10 \mu \mathrm{g} / \mathrm{mL}$. For infection with H-1PV, a MOI of $20 \mathrm{PFU} /$ cell with 5 days incubation period was chosen, due to selected experiments. ${ }^{6,79,22}$ DCs were isolated as described and seeded in 6-well plates in a ratio of 5:1 with SW480 at day 6 and cultivated for 3 days. Cells were harvested and stained with anti-CD80,-CD83 and -CD86 antibodies (BD Biosciences, San Jose, CA, USA). Expressions of these receptors were analyzed by FACScan ${ }^{\mathrm{TM}}$.

\section{Cytokine analysis via enzyme-linked immunosorbent assay (ELISA)}

The supernatant of cocultivated cells was collected before harvesting and storage at $-80^{\circ} \mathrm{C}$. Cytokine analyses of interferon gamma (IFN- $\gamma$ ), IL-6, and TNF- $\alpha$ were performed per protocol of ELISA kits (IFN- $\gamma$-Kit; Affymetrix, Santa Clara, CA, USA; IL-6 and TNF- $\alpha-$ Kits; ImmunoTools $\mathrm{GmbH})$. Microtiter plates (Corning Incorporated, Corning,
NY, USA) were coated with coating buffer and incubated overnight. After washing, the plate was blocked with blocking buffer at room temperature. Standards and samples were transferred into the wells and incubated overnight. The wells were washed again and biotin conjugate was transferred. After incubation, the cells were washed and incubated for half an hour with streptavidin-horse radish peroxidase conjugate. After washing once again, TMB (3,3',5'5-tetramethylbenzidine; Sigma-Aldrich) substrate solution was added and incubated. The plate was read out in an ELISA reader at $450 \mathrm{~nm}$ and values of $570 \mathrm{~nm}$ were subtracted.

\section{Results}

\section{Influence of H-IPV, tremelimumab and cytostatic drugs on cell viability}

We measured the influence of H-1PV, tremelimumab, 5-fluorouracil, irinotecan, and oxaliplatin on SW480 cell viability using an MTT assay.

After infection for 5 days, H-1PV reduced SW480 cell viability in an MOI-dependent manner. An MOI of 20 decreased viability to approximately $40 \%$ (cell control $=100 \%$ viability) (Figure 1$)$. Likewise, treatment with 5-fluorouracil, oxaliplatin, and irinotecan also resulted in time- and concentration-dependent reduction of SW480 cell viability (Figure 2).

Titration of tremelimumab up to a concentration of $240 \mu \mathrm{g} / \mathrm{mL}$ showed no direct influence on SW480 cell viability in the MTT assay (Figure 3). The same holds true for prolongation of incubation time. We next measured incubation time and the concentration-dependent influence of tremelimumab on different colorectal cancer cell lines. Cell viability of Caco-2, HCT116, and HT29 was not

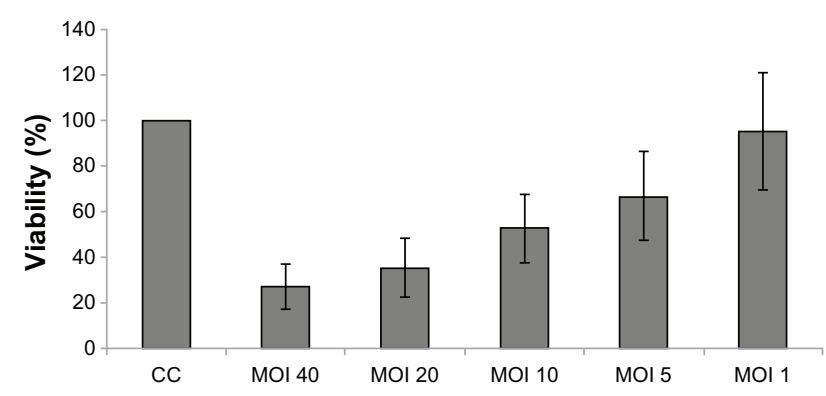

Figure I Influence of $\mathrm{H}$-IPV on SW480 viability.

Notes: Viability was measured by MTT assay; the effect of H-IPV infection on the viability of SW480 cells was measured by the MTT assay and expressed as the percentage of living cells in virus-infected cells versus control.

Abbreviations: CC, cell control; MOI, multiplicity of infection; MTT, 3-(4,5dimethylthiazol-2-yl)-2,5-diphenyltetrazolium bromide; $\mathrm{H}$-IPV, parvovirus $\mathrm{H}-\mathrm{I}$. 


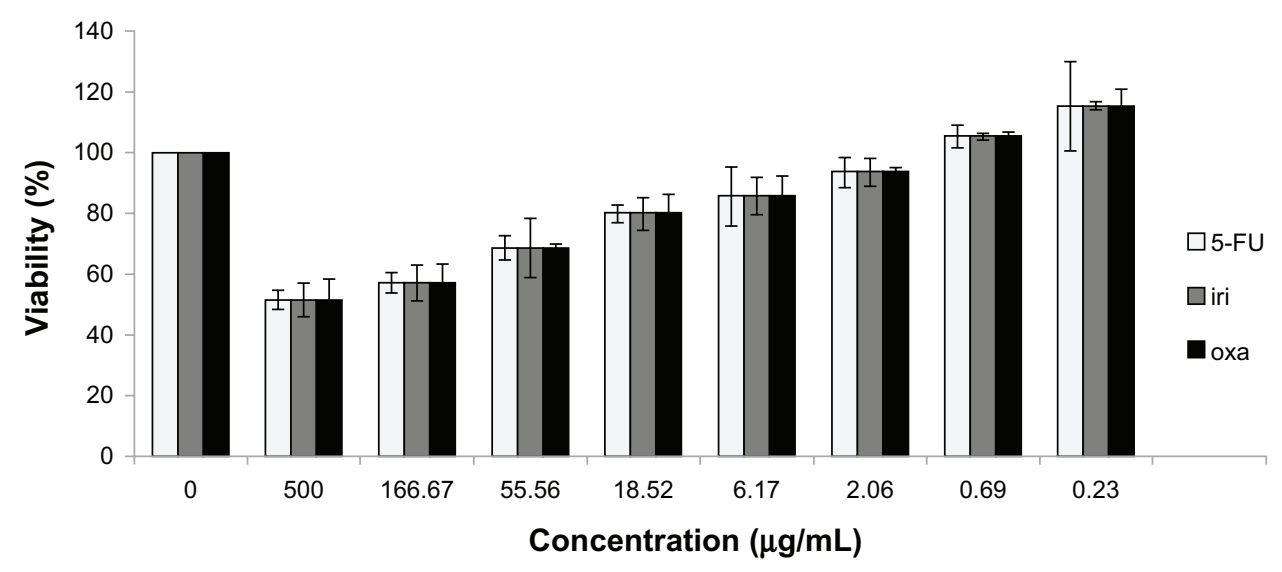

Figure 2 Influence of cytostatic drugs on SW480 cell viability.

Notes: Viability was measured by MTT assay. The effect of 5-FU, iri and oxa on the viability of SW480 cells, with an incubation period of 48 hours, was measured by MTT assay and expressed as the percentage of living cells in cells treated with different concentrations versus untreated cells $(0 \mu \mathrm{g} / \mathrm{mL})$.

Abbreviations: 5-FU, 5-fluorouracil; iri, irinotecan; oxa, oxaliplatin; MTT, 3-(4,5-dimethylthiazol-2-yl)-2,5-diphenyltetrazolium bromide.

influenced by tremelimumab as measured by MTT assay (data not shown).

\section{Expression of CTLA-4 after H-IPV infection or treatment with cytostatic drugs}

In order to treat colorectal cancer cell lines with anti-CTLA-4 antibody tremelimumab, we first investigated the expression of the target CTLA-4 intracellularly as well as on the cell surface. We used Caco-2 cells as a reference, which were previously demonstrated to express CTLA- $4 .{ }^{13}$ Influence of H-1PV and cytostatic drugs on CTLA-4 expression was measured by FACScan ${ }^{\mathrm{TM}}$.

SW480 expression of CTLA-4 exceeded expression of the positive control: Caco-2 cells. Treatment with 5-FU, oxaliplatin, or irinotecan resulted in increased extracellular expression of CTLA-4 while the intracellular CTLA-4 expression remained rather constant. Likewise, H-1PV treatment increased expression of CTLA-4 extracellularly, but not intracellularly (Figure 4).

Results indicate that drug treatment as well as virus infection seems to enforce extracellular expression of CTLA-4.

\section{DC maturation in coculture model with SW480}

We next determined the influence on DC maturation by measuring extracellular markers of DC activation and maturation using coculture experiments with SW480. CD80 and CD86 are coligands of activated DCs, and CD83 is highly expressed on maturated DCs. ${ }^{17}$

As shown in Figure 5, cocultivation of SW480 with iDCs alone did not induce maturation of DCs. In contrast, infection

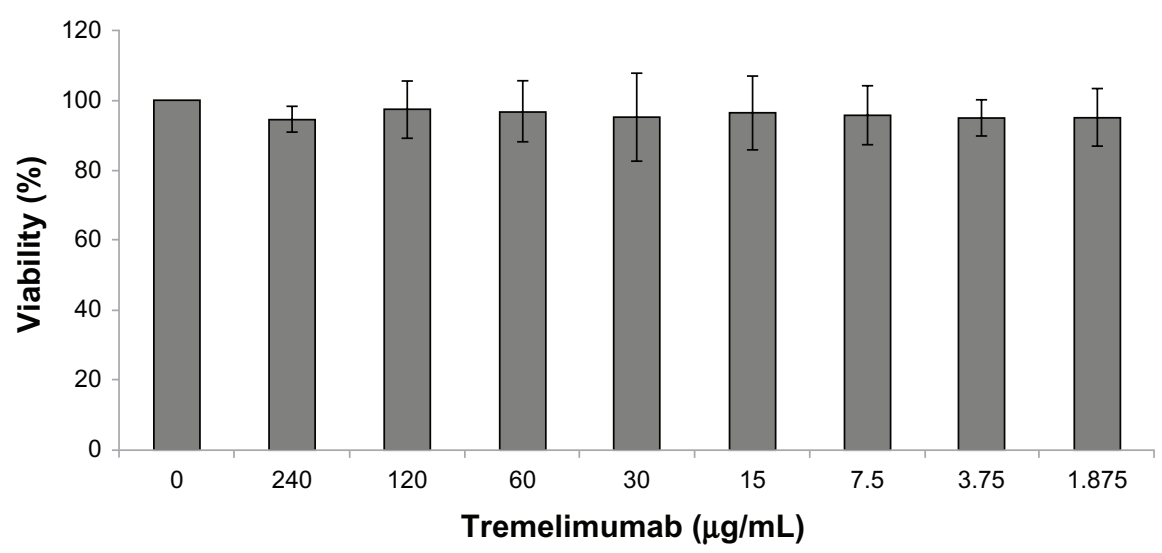

Figure 3 Influence of tremelimumab on SW480 cell viability.

Notes: The effect of anti-cytotoxic T-lymphocyte-associated antigen 4 antibody tremelimumab on the viability of SW480 cells, with an incubation period of 48 hours, was measured by MTT (3-(4,5-dimethylthiazol-2-yl)-2,5-diphenyltetrazolium bromide) assay and expressed as the percentage of living cells in tremelimumab treated cells versus untreated cells $(0 \mu \mathrm{g} / \mathrm{mL})$. 


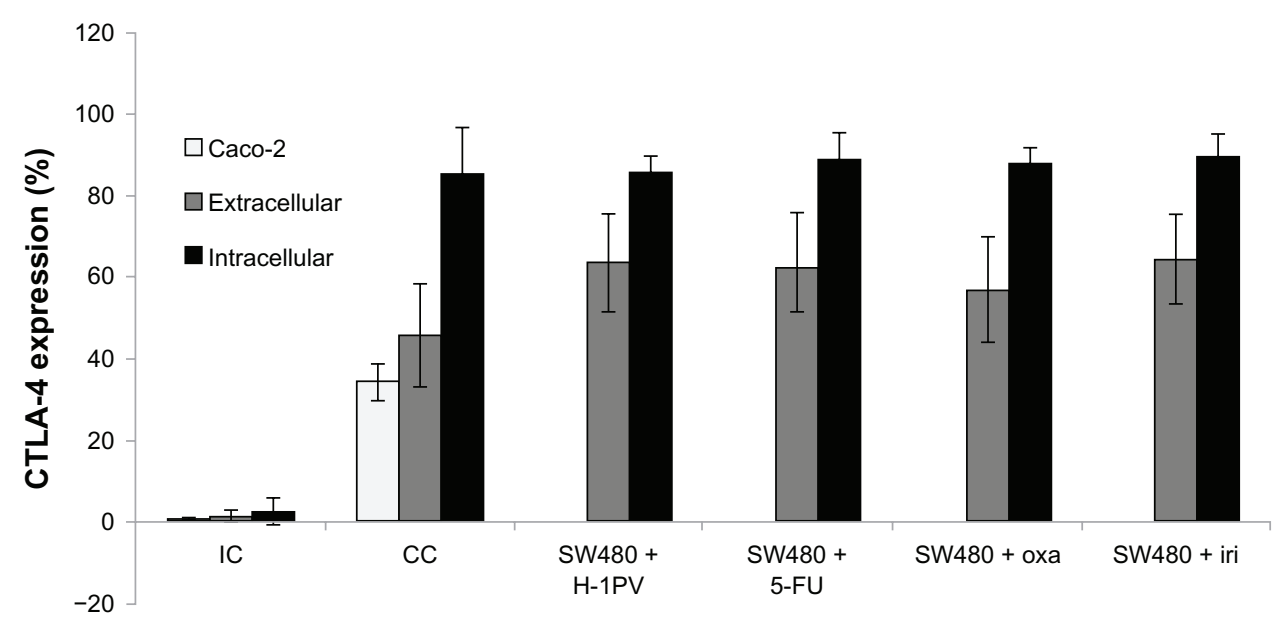

Figure 4 Intra- and extracellular expression of CTLA-4.

Notes: FACS analysis was used to verify the intracellular and extracellular expression of CTLA-4 in SW480 cells after H-IPV infection and treatment with cytostatic drugs. Caco-2 cells were used as positive control.

Abbreviations: CC, cell control; IC, isotypic control; $\mathrm{H}$-IPV, parvovirus $\mathrm{H}$-I; FACS, fluorescent activated cell sorting; CTLA-4, cytotoxic T-lymphocyte-associated antigen 4; 5-FU, 5-fluorouracil; iri, irinotecan; oxa, oxaliplatin.

of SW480 with H-1PV resulted in up to 20 times higher expression of activation and maturation markers. Among the cytostatic drugs we used, treatment with 5-fluorouracil was most efficient, resulting in a maturation of DCs to a point of extension almost comparable with $\mathrm{H}-1 \mathrm{PV}$ induction.
Irinotecan and especially oxaliplatin were clearly less potent than 5-fluorouracil or H-1PV in inducing maturation. Exclusive tremelimumab treatment, as well as treatment in combination with H-1PV or cytostatic drugs, showed no effect on DC maturation.

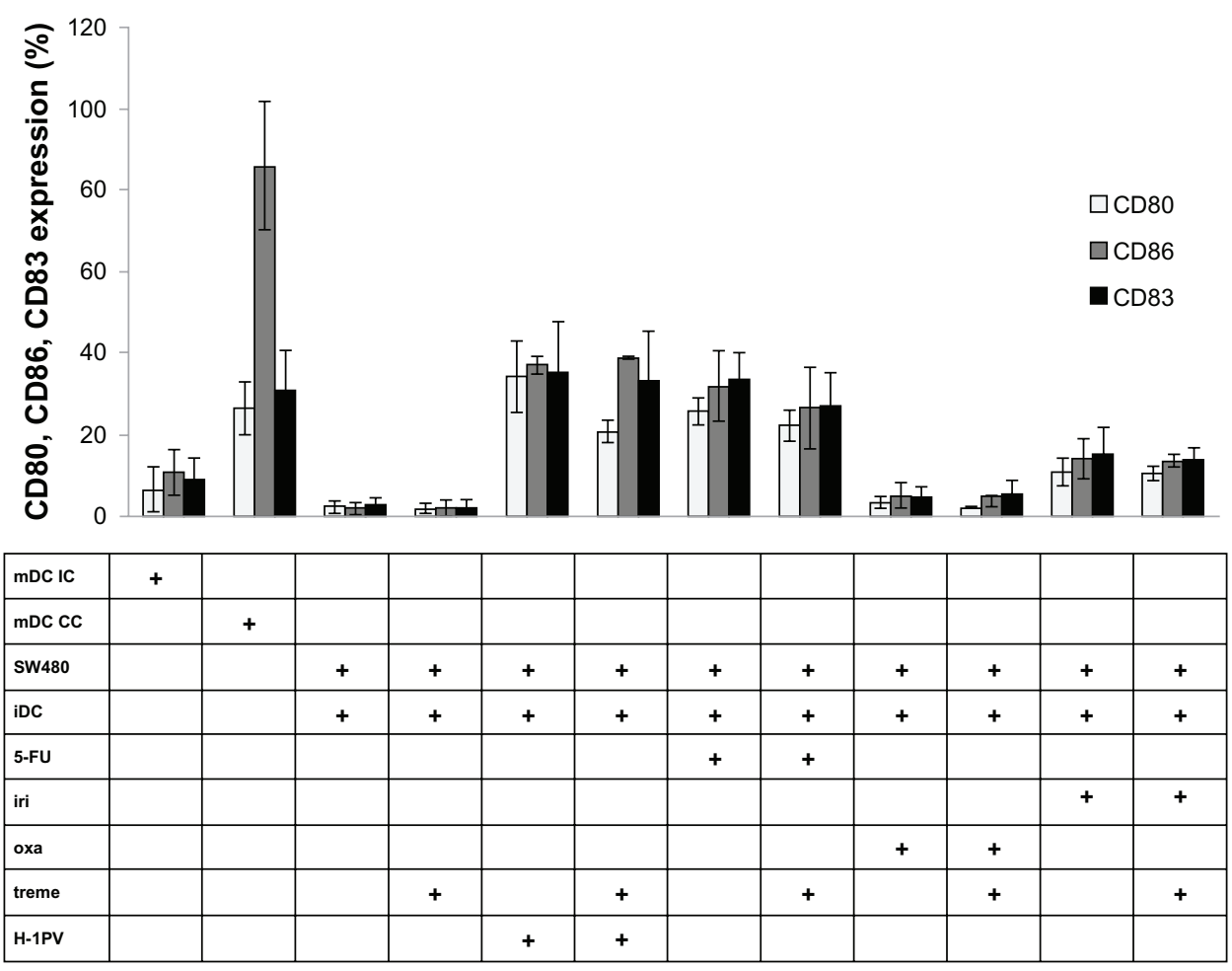

Figure 5 Analysis of CD80, CD86, CD83 expression.

Notes: Detection of CD80, CD86 and CD83 expression by FACS analysis in human DCs after co-incubation with H-IPV-infected or cytostatic drug treated and/or tremelimumab treated SW480 cells.

Abbreviations: 5-FU, 5-fluorouracil; CC, cell control; IC, isotypic control; iDC, immature DC; iri, irinotecan; mDC, mature DC; oxa, oxaliplatin; treme, tremelimumab; DC, dendritic cell; $\mathrm{H}-\mathrm{IPV}$, parvovirus $\mathrm{H}-\mathrm{I}$; CD, cluster of differentiation; FACS, fluorescent activated cell sorting. 


\section{Cytokine analysis}

In order to investigate the effects of cytokine levels on DC maturation, we performed ELISA measurements for cytokines IFN$\gamma$, TNF- $\alpha$, and IL-6 (Figure 6). TNF- $\alpha$ and IL-6 were part of the cytokine cocktail in a concentration of $0.01 \mu \mathrm{g} / \mathrm{mL}$ to promote maturation of iDCs. In the supernatant of mDCs, these cytokines showed a concentration that was almost 20 times higher, thus confirming mDCs as a positive control. The collected supernatants of cocultivated cells infected with H-1PV showed higher levels of IFN- $\gamma$, TNF- $\alpha$, and IL- 6 compared to noninfected cells, and cells treated with cytostatic drugs. The measured cytokine concentrations clearly exceeded those detected in the supernatant of $\mathrm{mDCs}$ by more than four times.

Tremelimumab treatment alone did not result in higher cytokine levels. This also holds true for cells treated with cytostatic drugs, where we could not detect an increase in cytokine concentrations. However, when we added tremelimumab to iDC cells coincubated with H-1PV-infected cells, a trend towards a higher IFN- $\gamma$ level under the influence of tremelimumab was detected; but, with a $P$-value of 0.198 , it was not statistically significant.

\section{Discussion}

It has been shown recently that colon carcinoma cell lines can efficiently be infected by $\mathrm{H}-1 \mathrm{PV}^{6-8}$ Our experiments indicate an MOI-dependent influence on cell viability and therefore demonstrate a concentration-dependent destruction of colorectal cancer cells. As parvoviruses showed a preference for multiplying in malignant-transformed cells, and cytotoxicity was selective for tumor cells, ${ }^{5}$ this indicates the suitability of this approach for colorectal cancer treatment. Additionally, and in line with previous experiments for other cell types, ${ }^{6,7,22}$ our coculture experiments demonstrate that DCs did not suffer from the presence of $\mathrm{H}-1 \mathrm{PV}$-infected colorectal cancer cells. Having defined the functionality of the system, we next analyzed the immune stimulatory effects of our colorectal cancer model system.

DCs showed a high expression of activation and maturation markers in the presence of infected SW480 cells, which was described for melanoma, ${ }^{6,7,22}$ but not for colorectal cancer.

IFN- $\gamma$ is a central mediator in the immune reaction by activating phagocytosis. ${ }^{23,24}$ Increased production of IFN- $\gamma$ was detected with H-1PV treated SW480 in coculture with iDCs. Additionally, and to a much larger extent, the coculture system increased the production of TNF- $\alpha$ and IL-6. These results confirm promising features of $\mathrm{H}-1 \mathrm{PV}$, which was described to improve antitumor immune response. ${ }^{5}$

In a model using human embryonic kidney cells (HEK293), H-1PV was found to exert its immunogenic

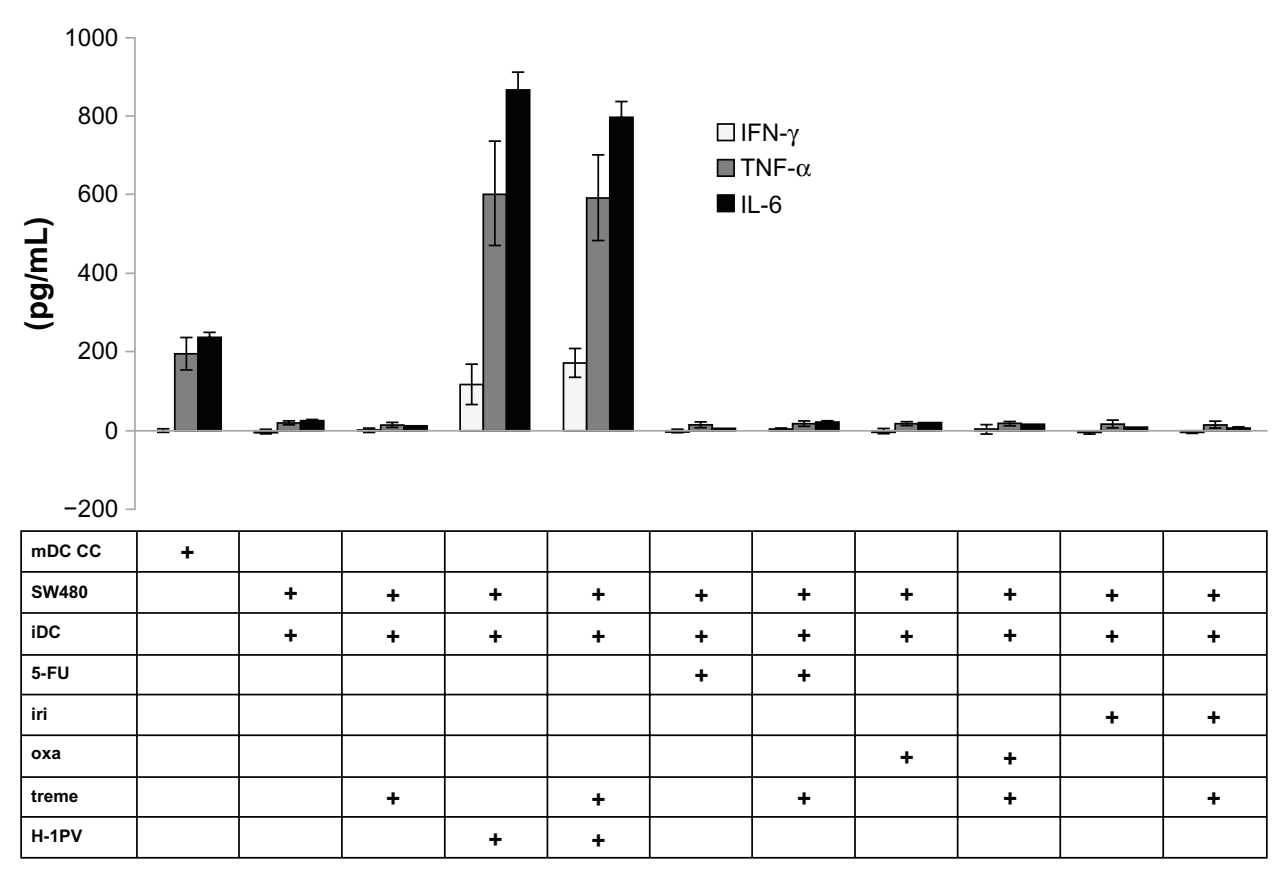

Figure 6 Cytokine analysis.

Notes: IFN- $\gamma$, TNF- $\alpha$ and IL-6 levels were measured by ELISA; supernatants of co-incubated DCs with H-IPV-infected and/or cytostatic drug treated and/or tremelimumab treated SW480 cells were analyzed by ELISA to detect cytokine levels. Cytokine production of treated cells was compared to mDC production of cytokines.

Abbreviations: 5-FU, 5-fluorouracil; CC, cell control; iDC, immature DC; iri, irinotecan; mDC, mature DC; oxa, oxaliplatin; treme, tremelimumab; DC, dendritic cell; IFN- $\gamma$, interferon gamma; TNF- $\alpha$, tumor necrosis factor alpha; IL-6, interleukin 6; ELISA, enzyme-linked immunosorbent assay; H-IPV, parvovirus $H$-I. 
effects through activation of toll-like receptor (TLR) 3 and TLR9, leading to enhanced nuclear factor kappa-lightchain-enhancer of activated B cells (NFKB) expression. Furthermore, in H-1PV-infected tumor cells lysates, TLR activation led to DC maturation and immune stimulation. ${ }^{25}$ Activation of DCs to generate a DC-based cancer immunotherapy was part of several investigations; ${ }^{26,27}$ especially, the idea of a DC based vaccination against cancer was of interest. ${ }^{28}$ With H-1PV, a potential candidate to gain DC maturation and activation was described. ${ }^{9}$ However, immune escape of tumor cells is still a problem to overcome. Expression of CTLA-4 on tumors was shown to be part of these escaping mechanisms. ${ }^{29}$ CTLA-4-expressing cells can capture costimulatory ligands like CD80 or CD86 via trans-endocytosis, leading to degradation inside the cells. ${ }^{30}$ To improve the antitumor immune response, the idea of combining H-1PV with tremelimumab, a CTLA-4-blocking monoclonal antibody, was generated.

Our experiments demonstrated a higher extracellular expression of CTLA-4 on H-1PV-infected SW480 cells, comparable to that induced by 5-FU, oxaliplatin, or irinotecan, than on non-treated cell control. We tested the hypothesis that blocking CTLA-4 using tremelimumab will induce improvement of activation, stimulation, and maturation of $\mathrm{DCs}^{31,32}$ in colorectal cancer cells. Coculture experiments showed no influence of tremelimumab on DC maturation for colon carcinoma cells, while a direct influence of tremelimumab on cell viability was excluded. This is in line with results from clinical Phase II trials where tremelimumab did not demonstrate a clinically meaningful single agent activity in patients with refractory metastatic colorectal cancer. ${ }^{33}$ In comparable receptor systems associated with the immune system like PD-1, blockade of receptor PD-1 or ligand PD-1 L did not gain objective responses for patients with colorectal cancer. ${ }^{34,35}$ However, combination therapies of tremelimumab with H-1PV or cytostatic drugs have not been evaluated thus far. In our setting, tremelimumab did not strengthen effects of H-1PV or cytostatic drugs on DC maturation. Nevertheless, influences on other parts of the immune system have to be investigated for these combinations. Blocking CTLA-4 is a potential stimulus of the immune system. Controlling and blocking of CTLA-4 proved to be important in mediation of viral infection and improving viral elimination of hepatitis B, C, and E. ${ }^{36-39}$ It was demonstrated that CTLA-4 blockade was associated with a decrease in immunosuppressive molecules such as indoleamine 2,3-dioxygenase or tumor growth factor- $\beta .{ }^{40}$ Also, regulatory T cells express CTLA-4 and were described to be important in the immune escape of tumors, and blocking of CTLA-4 resulted in autoimmunity and improved immune response. ${ }^{41}$ Blocking CTLA-4 and eliminating regulatory $\mathrm{T}$ cells showed synergistic effects. ${ }^{42}$ The mechanisms of CTLA-4 and its effector molecules, like indoleamine 2,3-dioxygenase, and also their interaction with regulatory $\mathrm{T}$ cells have been analyzed, ${ }^{41-43}$ but its influence on and in combination with oncolytic viruses like H-1PV remains to be elucidated. This confirms the necessity of investigating these mechanisms especially to explore the potential benefit of $\mathrm{H}-1 \mathrm{PV}$ by combination therapy with anti-CTLA-4 antibodies. Here, DC maturation was not improved by tremelimumab, but cytokine analysis showed a higher expression of IFN- $\gamma$ in cases of tremelimumab treated coculture. IFN- $\gamma$ increases expression of class I major histocompatibility complex as well as class II major histocompatibility complex on antigen presenting cells and is important for differentiation of $\mathrm{T}_{\mathrm{h}}-1$ cells. ${ }^{44}$ Higher levels of IFN- $\gamma$ were previously shown in supernatants of cytotoxic $\mathrm{T}$ lymphocytes in combination with $\mathrm{H}-1 \mathrm{PV}$-infected melanoma cell clones compared to noninfected cells. ${ }^{9}$ Also, combination of H-1PV-infected cells and tremelimumab treatment showed higher cytokine concentrations of TNF- $\alpha$ and IL- 6 compared to $\mathrm{mDC}$ cell control. TNF- $\alpha$ and IL-6 have synergistic effects on cancer; in particular by inducing apoptosis, and perhaps vascular targeting. ${ }^{45}$ High cytokine levels of both IFN- $\gamma$ and TNF- $\alpha$ are of special interest because of synergistic effects in cases of apoptosis. ${ }^{46,47}$ TNF- $\alpha$ is part of the $T_{h}-1$ cell immune response and is important for immune stimulating cytokine production, but was also described to gain a higher expression of the CTLA-4-similar surface molecule PD-1 on monocytes. ${ }^{48}$ Inhibiting this pathway resulted in a better immune response. ${ }^{48}$ IL-6 also inhibits regulatory $\mathrm{T}$ cells, which are inhibitors for the antitumor immune response, and several experiments with cytokine-induced killer cells showed a positive effect of high IL-6 levels on antitumor response. ${ }^{44,49}$ This is similar to blocking of CTLA-4 as described above, so combination of these two effects is of interest. Higher levels of TNF- $\alpha$ and IL- 6 were previously measured in a setting of cytotoxic $\mathrm{T}$ lymphocytes combined with iDCs and H-1PV-infected melanoma cells. ${ }^{7}$ IL-6 is also important in cases of vaccination models, as IL-6 provided by antigen-presenting cells is dispensable for proper $\mathrm{CD}^{+} \mathrm{T}$ cell memory generation. ${ }^{50} \mathrm{DC}$ vaccination in combination with CTLA-4 blockade was described to be successful in cases of peptide-pulsed DCs and combination with tremelimumab. ${ }^{51}$ Actual trials of oncolytic and immunotherapeutic vaccine virus JX-594 showed successfully that this virus can also be effective in cases of hepatocellular carcinoma by promoting 
lysis of tumor cells, activating an antivascular effect, and inducing a durable immune response. ${ }^{52}$ So, the idea of vaccination also for colorectal cancer should be further investigated and the combination of H-1PV and tremelimumab seems to be a promising candidate.

\section{Conclusion}

H-1PV showed multiple features that make it a promising candidate for further investigations in colorectal cancer. Forthcoming results of clinical trials with $\mathrm{H}-1 \mathrm{PV}$, for example, in cases of glioblastoma multiformes, are being awaited with interest ${ }^{53}$ and the latest trials have demonstrated the potential of oncolytic viruses in the case of hepatocellular carcinoma. ${ }^{52}$ Furthermore, colorectal cancer proved to be a promising entity for additional investigations of immunotherapy. Here, the potential of H-1PV for generating an antitumor immune reaction via DC maturation also for SW480 cells is of special interest. Moreover, the combination of tremelimumab with H-1PV and their effects on the immune system need further investigation as CTLA-4 is part of the tumor immune escape mechanism and plays an important role in the mediation of the human immune system. It needs to be understood in detail in order to improve cancer immunotherapy.

\section{Acknowledgment}

Aspects of this article are part of the doctoral thesis of B Heinrich.

\section{Disclosure}

The authors report no conflicts of interest in this work.

\section{References}

1. Mazzolini G, Murillo O, Atorrasagasti C, et al. Immunotherapy and immunoescape in colorectal cancer. World J Gastroenterol. 2007;13(44): 5822-5831.

2. Blattman JN, Greenberg PD. Cancer immunotherapy: a treatment for the masses. Science. 2004;305(5681):200-205.

3. Mace AT, Harrow SJ, Ganly I, Brown SM. Cytotoxic effects of the oncolytic herpes simplex virus HSV1716 alone and in combination with cisplatin in head and neck squamous cell carcinoma. Acta Otolaryngol. 2007;127(8):880-887.

4. Chalikonda S, Kivlen MH, O'Malley ME, et al. Oncolytic virotherapy for ovarian carcinomatosis using a replication-selective vaccinia virus armed with a yeast cytosine deaminase gene. Cancer Gene Ther. 2008;15(2): $115-125$.

5. Rommelaere J, Geletneky K, Angelova AL, et al. Oncolytic parvoviruses as cancer therapeutics. Cytokine Growth Factor Rev. 2010;21(2-3):185-195.

6. Moehler M, Blechacz B, Weiskopf N, et al. Effective infection, apoptotic cell killing and gene transfer of human hepatoma cells but not primary hepatocytes by parvovirus $\mathrm{H} 1$ and derived vectors. Cancer Gene Ther. 2001;8(3):158-167.
7. Moehler M, Sieben M, Roth S, et al. Activation of the human immune system by chemotherapeutic or targeted agents combined with the oncolytic parvovirus H-1. BMC Cancer. 2011;11:464.

8. Malerba M, Daeffler L, Rommelaere J, Iggo RD. Replicating parvoviruses that target colon cancer cells. J Virol. 2003;77(12): 6683-6691.

9. Moehler MH, Zeidler M, Wilsberg V, et al. Parvovirus H-1-induced tumor cell death enhances human immune response in vitro via increased phagocytosis, maturation, and cross-presentation by dendritic cells. Hum Gene Ther. 2005;16(8):996-1005.

10. van Mierlo GJ, Boonman ZF, Dumortier HM, et al. Activation of dendritic cells that cross-present tumor-derived antigen licenses CD8+ CTL to cause tumor eradication. J Immunol. 2004;173(11):6753-6759.

11. Chan CW, Housseau F. The 'kiss of death' by dendritic cells to cancer cells. Cell Death Differ. 2008;15(1):58-69.

12. Wang XB, Fan ZZ, Anton D, et al. CTLA4 is expressed on mature dendritic cells derived from human monocytes and influences their maturation and antigen presentation. BMC Immunol. 2011;12:21.

13. Contardi E, Palmisano GL, Tazzari PL, et al. CTLA-4 is constitutively expressed on tumor cells and can trigger apoptosis upon ligand interaction. Int J Cancer. 2005;117(4):538-550.

14. Alegre ML, Frauwirth KA, Thompson CB. T-cell regulation by CD28 and CTLA-4. Nat Rev Immunol. 2001;1(3):220-228.

15. Ribas A. Tumor immunotherapy directed at PD-1. N Engl J Med. 2012;366(26):2517-2519.

16. Ribas A, Hanson DC, Noe DA, et al. Tremelimumab (CP-675,206), a cytotoxic $\mathrm{T}$ lymphocyte associated antigen 4 blocking monoclonal antibody in clinical development for patients with cancer. Oncologist. 2007;12(7):873-883.

17. Poirier N, Blancho G, Vanhove B. A more selective costimulatory blockade of the CD28-B7 pathway. Transpl Int. 2011;24(1):2-11.

18. Ribas A. Overcoming immunologic tolerance to melanoma: targeting CTLA-4 with tremelimumab (CP-675,206). Oncologist. 2008; 13 Suppl 4:10-15.

19. Ascierto PA, Marincola FM, Ribas A. Anti-CTLA4 monoclonal antibodies: the past and the future in clinical application. JTransl Med. 2011;9:196.

20. Oderup C, Cederbom L, Makowska A, Cilio CM, Ivars F. Cytotoxic $\mathrm{T}$ lymphocyte antigen-4-dependent down-modulation of costimulatory molecules on dendritic cells in CD4+CD25+ regulatory T-cell-mediated suppression. Immunology. 2006;118(2):240-249.

21. Zhu Q, Liu JY, Yang CM, et al. Influence of antitumor drugs on the expression of Fas system in SW480 colon cancer cells. Eur J Gastroenterol Hepatol. 2006;18(10):1071-1077.

22. Moehler M, Zeidler M, Schede J, et al. Oncolytic parvovirus H1 induces release of heat-shock protein HSP72 in susceptible human tumor cells but may not affect primary immune cells. Cancer Gene Ther. 2003;10(6):477-480.

23. Billiau A. Interferon-gamma: biology and role in pathogenesis. $A d v$ Immunol. 1996;62:61-130.

24. Isaacs A, Lindenmann J. Virus interference. I. The interferon. Proc $R$ Soc Lond B Biol Sci. 1957;147(927):258-267.

25. Sieben M, Schäfer P, Dinsart C, Galle PR, Moehler M. Activation of the human immune system via toll-like receptors by the oncolytic parvovirus H-1. Int J Cancer. 2013;132(11):2548-2556.

26. Nencioni A, Brossart P. New perspectives in dendritic cell-based cancer immunotherapy. BioDrugs. 2001;15(10):667-679.

27. Ballestrero A, Boy D, Moran E, Cirmena G, Brossart P, Nencioni A. Immunotherapy with dendritic cells for cancer. Adv Drug Deliv Rev. 2008;60(2):173-183.

28. Koido S, Hara E, Homma S, Fujise K, Gong J, Tajiri H. Dendritic/tumor fusion cell-based vaccination against cancer. Arch Immunol Ther Exp (Warsz). 2007;55(5):281-287.

29. Iida T, Ohno H, Nakaseko C, et al. Regulation of cell surface expression of CTLA-4 by secretion of CTLA-4-containing lysosomes upon activation of CD4+ T cells. J Immunol. 2000;165(9):5062-5068. 
30. Qureshi OS, Zheng Y, Nakamura K, et al. Trans-endocytosis of CD80 and CD86: a molecular basis for the cell-extrinsic function of CTLA-4. Science. 2011;332(6029):600-603.

31. Korman AJ, Peggs KS, Allison JP. Checkpoint blockade in cancer immunotherapy. Adv Immunol. 2006;90:297-339.

32. Wallet MA, Sen P, Tisch R. Immunoregulation of dendritic cells. Clin Med Res. 2005;3(3):166-175.

33. Chung KY, Gore I, Fong L, et al. Phase II study of the anti-cytotoxic T-lymphocyte-associated antigen 4 monoclonal antibody, tremelimumab, in patients with refractory metastatic colorectal cancer. J Clin Oncol. 2010;28(21):3485-3490.

34. Topalian SL, Hodi FS, Brahmer JR, et al. Safety, activity, and immune correlates of anti-PD-1 antibody in cancer. $N$ Engl J Med. 2012;366(26):2443-2454.

35. Brahmer JR, Tykodi SS, Chow LQ, et al. Safety and activity of antiPD-L1 antibody in patients with advanced cancer. $N$ Engl J Med. 2012;366(26):2455-2465.

36. Schurich A, Khanna P, Lopes AR, et al. Role of the coinhibitory receptor cytotoxic T lymphocyte antigen-4 on apoptosis-Prone CD8 T cells in persistent hepatitis B virus infection. Hepatology. 2011;53(5): 1494-1503.

37. Yin $\mathrm{Y}, \mathrm{Wu} \mathrm{C}$, Song J, et al. DNA immunization with fusion of CTLA-4 to hepatitis B virus (HBV) core protein enhanced Th2 type responses and cleared $\mathrm{HBV}$ with an accelerated kinetic. PLoS One. 2011;6(7):e22524.

38. Suneetha PV, Pischke S, Schlaphoff V, et al. Hepatitis E virus (HEV)specific T-cell responses are associated with control of HEV infection. Hepatology. 2012;55(3):695-708.

39. Raziorrouh B, Ulsenheimer A, Schraut W, et al. Inhibitory molecules that regulate expansion and restoration of HCV-specific CD4+ T cells in patients with chronic infection. Gastroenterology. 2011;141(4):14221431,1431 e1-6.

40. Hryniewicz A, Boasso A, Edghill-Smith Y, et al. CTLA-4 blockade decreases TGF-beta, IDO, and viral RNA expression in tissues of SIVmac251-infected macaques. Blood. 2006;108(12):3834-3842.

41. von Euw E, Chodon T, Attar N, et al. CTLA4 blockade increases Th17 cells in patients with metastatic melanoma. J Transl Med. 2009;7:35
42. Suarez N, Alfaro C, Dubrot J, et al. Synergistic effects of CTLA-4 blockade with tremelimumab and elimination of regulatory $\mathrm{T}$ lymphocytes in vitro and in vivo. Int J Cancer. 2011;129(2):374-386.

43. Katz JB, Muller AJ, Prendergast GC. Indoleamine 2,3-dioxygenase in T-cell tolerance and tumoral immune escape. Immunol Rev. 2008;222:206-221.

44. Schroder K, Hertzog PJ, Ravasi T, Hume DA. Interferon-gamma: an overview of signals, mechanisms and functions. J Leukoc Biol. 2004;75(2):163-189.

45. van Horssen R, Ten Hagen TL, Eggermont AM. TNF-alpha in cancer treatment: molecular insights, antitumor effects, and clinical utility. Oncologist. 2006;11(4):397-408.

46. Fulda S, Debatin KM. Signaling through death receptors in cancer therapy. Curr Opin Pharmacol. 2004;4(4):327-332.

47. Fulda S, Debatin KM. Death receptor signaling in cancer therapy. Curr Med Chem Anticancer Agents. 2003;3(4):253-262.

48. Said EA, Dupuy FP, Trautmann L, et al. Programmed death-1-induced interleukin-10 production by monocytes impairs CD4+ T cell activation during HIV infection. Nat Med. 2010;16(4):452-459.

49. Gajewski TF, Fitch FW. Anti-proliferative effect of IFN-gamma in immune regulation. I. IFN-gamma inhibits the proliferation of Th2 but not Th1 murine helper T lymphocyte clones. J Immunol. 1988;140(12): $4245-4252$.

50. Daudelin JF, Mathieu M, Boulet S, Labrecque N. IL-6 production by dendritic cells is dispensable for CD8(+) memory T-cell generation. Biomed Res Int. 2013;2013:126189.

51. Ribas A, Comin-Anduix B, Chmielowski B, et al. Dendritic cell vaccination combined with CTLA4 blockade in patients with metastatic melanoma. Clin Cancer Res. 2009;15(19):6267-6276.

52. Heo J, Reid T, Ruo L, et al. Randomized dose-finding clinical trial of oncolytic immunotherapeutic vaccinia JX-594 in liver cancer. Nat Med. 2013;19(3):329-336.

53. Geletneky K, Huesing J, Rommelaere J, et al. Phase I/IIa study of intratumoral/intracerebral or intravenous/intracerebral administration of Parvovirus H-1 (ParvOryx) in patients with progressive primary or recurrent glioblastoma multiforme: ParvOryx01 protocol. BMC Cancer. 2012;12:99.
OncoTargets and Therapy

\section{Publish your work in this journal}

OncoTargets and Therapy is an international, peer-reviewed, open access journal focusing on the pathological basis of all cancers, potential targets for therapy and treatment protocols employed to improve the management of cancer patients. The journal also focuses on the impact of management programs and new therapeutic agents and protocols on

\section{Dovepress}

patient perspectives such as quality of life, adherence and satisfaction The manuscript management system is completely online and includes a very quick and fair peer-review system, which is all easy to use. Visit http://www.dovepress.com/testimonials.php to read real quotes from published authors. 